\title{
Perfil de morbidade pediátrica em um hospital público de ensino do Paraná
}

\author{
Profile of pediatric morbidity in a university teaching \\ hospital of Paraná
}

Amanda Khetleen Gussoㅎ Márcia Helena de Souza Freire ${ }^{2}$; Gabrielle Freitas Saganski ${ }^{3}$; Maythe Pacheco da Silva de Oliveira ${ }^{4}$; Sylvia Carriel Dias ${ }^{5} ;$ Michelle Thais Migoto ${ }^{6}$

1. Acadêmica de Enfermagem. Universidade Federal do Paraná (UFPR). Curitiba - Paraná- Brasil. ORCID: https://orcid.org/0000-0002-9887-9898. Email: amandakgusso@gmail.com

2. Enfermeira. Doutora em Saúde Pública. Docente do Departamento de Enfermagem da UFPR. Curitiba - Paraná - Brasil. ORCID: https://orcid.org/0000-0003-3941-3673.Email: marciahelenafreire@gmail. com

3. Enfermeira. Mestre em Enfermagem. Doutoranda no Programa de Pós-Graduação em Enfermagem da UFPR. Curitiba - Paraná - Brasil. ORCID: https://orcid.org/0000-0001-9716-659X.Email: gabisaga@ gmail.com

4. Enfermeira. Universidade Federal do Paraná (UFPR). Curitiba - Paraná - Brasil. ORCID: https://orcid. org/0000-0001-8873-7872.Email: maythepacheco@gmail.com

5. Acadêmica de Enfermagem. Universidade Federal do Paraná (UFPR). Curitiba - Paraná - Brasil. ORCID: https://orcid.org/0000-0002-6508-5602.Email: syl.carriel@gmail.com

6. Enfermeira. Mestre em Enfermagem. Doutoranda no Programa de Pós-Graduação em Enfermagem da UFPR. Curitiba - Paraná - Brasil. ORCID: https://orcid.org/0000-0002-8546-8694.Email: michellemigoto@gmail.com

CONTATO: Michelle Thais Migoto | Avenida Pref. Lothario Meissner, 632 - Bloco Didático || - $3^{\circ}$ andar | Jardim Botânico | Curitiba | Paraná | Brasil | Telefone: (41) 98760-0349 | Email: michellemigoto@gmail.com

COMO CITAR: Gusso AK; Freire MHS; Saganski GF; Oliveira MPS; Dias SC; Migoto MT. Perfil de morbidade pediátrica em um hospital público de ensino do Paraná. R. Saúde Públ. Paraná. 2020 Jul;3(1):51-61. 
RESUMO O objetivo foi caracterizar as internações ocorridas em um serviço clínico pediátrico, de um hospital de ensino de Curitiba, Paraná. Estudo quantitativo, transversal, descritivo, utilizou dados secundários do sistema de informação hospitalar da instituição em estudo, com recorte às internações de crianças e adolescentes na Clínica Pediátrica, entre 2015 e 2017 . Foram identificadas 491 internações no período, com proporções próximas entre os anos. A análise conclui: prevalência de internações do sexo feminino; em idade escolar; procedentes da $2^{a}$ Regional de Saúde do Paraná. Como causas das internações: neoplasias; doenças endócrinas, nutricionais e metabólicas; e, doenças do aparelho respiratório. Portanto, o perfil de morbidade contribui para a compreensão da transição epidemiológica. Conhecer as características das internações fornece subsídios para a gestão do serviço favorecendo a qualidade da assistência à criança.

PALAVRAS-CHAVES: Assistentes de Pediatria. Morbidade. Hospitalização. Perfil de Saúde. Enfermagem Pediátrica. Gestão em Saúde.

ABSTRACT The objective of this study was to characterize hospitalizations of a pediatric clinical service in a teaching hospital in Curitiba, state of Paraná. This is a quantitative, cross-sectional, descriptive study, using secondary data from the hospital information system, focusing on hospitalizations of children and adolescents at the Pediatric Clinic, between 2015 and 2017. There were 491 hospitalizations in the period, with similar proportions within the years. The analysis concludes: prevalence of female hospitalizations; school-age individuals; from 2nd Regional Health Unit of Paraná. The causes of hospitalizations: Neoplasms; Endocrine, Nutritional, Metabolic and Respiratory Tract Diseases. Therefore, the morbidity profile contributes to the understanding of the epidemiological transition. Knowing the characteristics of hospitalizations provides subsidies for service management, favoring the quality of childcare.

KEYWORDS: Pediatric Assistants. Morbidity. Hospitalization. Health Profile. Pediatric Nursing. Health Management.

\section{INTRODUÇÃO}

A assistência em Saúde da Criança compreende uma especialidade multiprofissional que atende o indivíduo desde o seu nascimento até o final da adolescência'. O Estatuto da Criança e do Adolescente (ECA), de 1990, considera como criança o indivíduo de até doze anos de idade incompletos². E o Sistema Único de Saúde (SUS) recomenda que os serviços de pediatria incluam o atendimento aos indivíduos menores de 16 anos $^{2}$, estendendo a idade assegurada pelo ECA.

Os estudos epidemiológicos sobre as internações hospitalares suscitam indícios de fragilidade evidenciando as condições do sistema de saúde, além de possibilitar a análise sobre as causas de internações, 
as quais favorecerão compreender seus determinantes locais e regionais ${ }^{3}$. Já a produção de evidências locais para a tomada de decisão, possibilita maior eficácia para a seleção das estratégias preventivas e terapêuticas em uma decisão clínica, embasando assim a elaboração de planos de atenção à saúde para prevenir o agravamento das doenças e evitar as internações hospitalares ${ }^{4-5}$.

Resultados de um estudo brasileiro confirmam haver características heterogêneas nas internações de crianças até quatro anos de idade, entre as regiões do país, registrando majoritariamente um agrupamento de doenças, apontadas como evitáveis na esfera da atenção básica, como as doenças do aparelho respiratório e as doenças infecciosas e parasitárias ${ }^{5}$. Destaca-se que há reduzido quantitativo de estudos que abordam caracterização da demanda dos serviços pediátricos hospitalares.

Considera-se que o desenvolvimento de estudos convergentes à caracterização das internações hospitalares de crianças e adolescentes poderá favorecer a formulação de evidências para a tomada de decisão pelos gestores do serviço, e contribuir para a avaliação regionalizada do sistema de saúde, considerando o fluxo entre os serviços de diferentes complexidades. Nesta consonância, buscou-se caracterizar o perfil de morbidade de um serviço clínico pediátrico, de hospital público de ensino, do estado do Paraná.

\section{MÉTODO}

Estudo quantitativo, transversal descritivo ${ }^{6}$, desenvolvido de acordo com as recomendações de estudos observacionais apresentadas na iniciativa Strengthening the Reporting of Observational Studies in Epidemiology (STROBE)?.

O cenário é parte de um hospital público de ensino, localizado em Curitiba, estado do Paraná, referência estadual para o atendimento de alto risco, como também para os estados vizinhos, vinculado a uma universidade pública e sob a cogestão da Empresa Brasileira de Serviços Hospitalares (EBSERH) ${ }^{8}$. A Clínica Pediátrica estudada disponibilizava no período de 2015 a 2017, seis leitos para internação de diversas especialidades, como: Endócrino; Neurologia; Pneumologia; Gastrologia; Hepatologia; Cardiologia; e Dermatologia. Esta compartilhava o mesmo espaço físico da Clínica de Hemato-oncologia Pediátrica, que contemplava mais seis leitos. A equipe multiprofissional deste serviço inclui médicos especializados, residentes de diversas áreas e programas, fisioterapeutas, nutricionistas, psicólogos, assistentes sociais, musicoterapeuta, recreacionista, copeiras e lactaristas. A equipe de enfermagem é composta por 14 técnicos de enfermagem, oito auxiliares de enfermagem e sete enfermeiros, dimensionados para a cobertura dos três turnos.

Foram utilizados dados secundários das internações de crianças e adolescentes, de 0 a 14 anos, importados do sistema de informação hospitalar (SIH) próprio da instituição, relativos ao período de 2015 a 2017. O sistema é gerenciado pelo Serviço de Informática local e tem capacidade para armazenamento dos dados de todos os atendimentos e procedimentos realizados. Vale ressaltar que o sistema possibilita a inclusão de apenas um (1) diagnóstico médico por linha, em cada internação identificada com nome, data e registro do atendimento, o que pode acarretar em repetição de linhas para a mesma internação que compõem o banco de dados. Para minimizar este viés de superestimação do número de internações, foi aplicado filtro para exclusão de internações que se repetiam, as quais foram verificadas pela exata convergência das informações de três campos, a saber: nome do paciente, registro de atendimento e data de internação. Este fato explica ser inferior o quantitativo total de internações quando comparado ao quantitativo total dos diagnósticos de internação, refletindo as situações nas quais o diagnóstico médico não foi único. 
As variáveis independentes, quantitativas e discretas, foram: sexo (masculino e feminino); faixa etária neonato (menores de 28 dias), lactente (de 28 dias a menor de 1 ano), pré-escolar (de 1 a 4), escolar (de 5 a 11) e, adolescente (de 12 até 19); tipo de alta (Transferência, Alta Médica, Óbito e Alta a pedido); procedência, segundo município ( $\mathrm{n}=399)$ e, Regional de Saúde $(\mathrm{n}=22)$ do estado do Paraná; e, Diagnósticos de Internação - somados o Principal Confirmado ao Principal suspeito, quando presente, evidenciando-se a Causa Básica da internação segundo a Classificação Internacional de Doenças e Problema Relacionados com a Saúde (CID$10)^{9}$.

A organização dos dados das variáveis foi realizada com o apoio do Microsoft Office Excel®, versão 2016. A análise transcorreu mediante estatística descritiva, do tipo univariada, aplicando-se medidas de localização ou tendência central, como: frequência, proporção e mediana, com apresentação tabular. Esta pesquisa foi aprovada pelos Comitês de Ética do Setor Saúde e do Complexo Hospital de Clínicas, sob parecer 1.061.330 e 1.032.347, respectivamente. Respeitando as recomendações da Resolução 466 de $2012^{10}$.

\section{RESULTADOS}

No período estudado houve 491 internações no serviço clínico pediátrico, sem diferenças significativas no quantitativo dos três anos. As características predominantes foram: sexo feminino (50,1\%); crianças em faixa etária escolar (41,3\%) e pré-escolar (28,1\%); com desfecho de alta médica (69,0\%) e, ausência de registro de evasão (Tabela 1).

Tabela 1 - Crianças e adolescentes internados segundo sexo, idade e tipo de alta, serviço clínico pediátrico de hospital público de ensino. Curitiba - PR, 2015 a 2017

\begin{tabular}{lcccccccc}
\hline \multirow{2}{*}{ Variáveis } & \multicolumn{2}{c}{$\mathbf{2 0 1 5}$} & \multicolumn{2}{c}{$\mathbf{2 0 1 6}$} & \multicolumn{2}{c}{$\mathbf{2 0 1 7}$} & \multicolumn{2}{c}{ Total } \\
\cline { 2 - 9 } & $\mathbf{n}$ & $\%$ & $\mathbf{n}$ & $\%$ & $\mathbf{n}$ & $\%$ & $\mathbf{n}$ & $\%$ \\
\hline Sexo & 166 & 100,00 & 164 & 100,00 & 161 & 100,0 & 491 & 100,0 \\
\hline Masculino & 82 & 49,4 & 87 & 53,0 & 76 & 47,2 & 245 & 49,9 \\
Feminino & 84 & 50,6 & 77 & 46,9 & 85 & 52,8 & 246 & 50,1 \\
\hline Idade & 166 & 100,0 & 164 & 100, & 161 & 100,0 & 491 & 100,0 \\
\hline Neonato & - & - & 1 & 0,6 & - & - & 1 & 0,2 \\
Lactente & 21 & 12,6 & 14 & 8,5 & 15 & 9,3 & 50 & 10,2 \\
Pré-escolar & 48 & 28,9 & 31 & 18,9 & 59 & 36,6 & 138 & 28,1 \\
Escolar & 65 & 39,1 & 80 & 48,8 & 58 & 36,0 & 203 & 41,3 \\
Adolescente & 32 & 19,30 & 38 & 23,1 & 29 & 18,0 & 99 & 20,1 \\
\hline Tipo de Alta & 166 & 100,0 & 164 & 100,0 & 161 & 100,0 & 491 & 100,0 \\
\hline Transferência & 53 & 31,9 & 51 & 31,1 & 46 & 28,6 & 150 & 30,5 \\
Alta Médica & 112 & 67,4 & 113 & 68,9 & 114 & 70,8 & 339 & 69,0 \\
Óbito & 1 & 0,6 & - & - & - & - & 1 & 0,2 \\
Alta a pedido & - & - & - & - & 1 & 0,6 & 1 & 0,2 \\
\hline
\end{tabular}

Fonte: Dados disponibilizados pelo Sistema de Informação Hospitalar. Elaborado pelos autores (2018). 
Na Tabela 2, evidencia-se que quase a totalidade dos pacientes pediátricos procederam da $2^{\text {a }}$ Regional de Saúde - Metropolitana (61\%), que é composta por 29 municípios e tem como sede o município de Curitiba. Esta inclui a Região Metropolitana da capital do estado, na qual se encontra o hospital do estudo. Ademais, nos três anos contemplados ocorreram internações de crianças procedentes de outros estados, como Tocantins e Santa Catarina.

Tabela 2 - Procedência das crianças e adolescentes internados, serviço clínico pediátrico de hospital público de ensino. Curitiba - PR, 2015 $-2017$.

\begin{tabular}{|c|c|c|c|c|c|c|c|c|}
\hline \multirow{2}{*}{ Procedência } & \multicolumn{2}{|c|}{2015} & \multicolumn{2}{|c|}{2016} & \multicolumn{2}{|c|}{2017} & \multicolumn{2}{|c|}{ Total } \\
\hline & $\mathrm{n}$ & $\%$ & $\mathbf{n}$ & $\%$ & $\mathbf{n}$ & $\%$ & $\mathbf{n}$ & $\%$ \\
\hline Número Internações & 166 & 100,0 & 164 & 100,0 & 161 & 100,0 & 491 & 100,0 \\
\hline Paraná & 164 & 98,8 & 161 & 98,1 & 156 & 96.9 & 481 & 97,9 \\
\hline Outros estados & 2 & 1,2 & 3 & 1,8 & 5 & 3,1 & 10 & 2,0 \\
\hline $\begin{array}{l}\mathrm{RS}^{*} \text { do Paraná e } \\
\text { município-sede }\end{array}$ & 164 & 100,0 & 161 & 100,0 & 156 & 100,0 & 481 & 100,0 \\
\hline $1^{a}$ Paranaguá & 14 & 8,5 & 9 & 5,6 & 7 & 4,5 & 30 & 6,2 \\
\hline $2^{\mathrm{a}}$ Curitiba & 101 & 61,6 & 102 & 63,3 & 95 & 60,9 & 298 & 61,9 \\
\hline $3^{a}$ Ponta Grossa & 2 & 1,2 & 2 & 1,2 & 6 & 3,8 & 10 & 2,1 \\
\hline $4^{a}$ Irati & 5 & 3,0 & 6 & 3,7 & 2 & 1,3 & 13 & 2,7 \\
\hline $5^{\mathrm{a}}$ Guarapuava & 6 & 3,6 & 1 & 0,6 & 6 & 3,8 & 13 & 2,7 \\
\hline $6^{a}$ União da Vitória & 1 & 0,6 & 3 & 1,8 & 2 & 1,3 & 6 & 1,2 \\
\hline $7^{\mathrm{a}}$ Pato Branco & 1 & 0,6 & 2 & 1,2 & 5 & 3,2 & 8 & 1,6 \\
\hline $8^{a}$ Francisco Beltrão & 2 & 1,2 & - & - & 1 & 0,6 & 3 & 0,6 \\
\hline $9^{a}$ Foz do Iguaçu & 3 & 1,8 & 1 & 0,6 & - & - & 4 & 0,8 \\
\hline $10^{a}$ Cascavel & 2 & 1,2 & 1 & 0,6 & - & - & 3 & 0,8 \\
\hline $11^{\mathrm{a}}$ Campo Mourão & 7 & 4,2 & 13 & 8,0 & 12 & 7,7 & 32 & 6.6 \\
\hline $12^{\mathrm{a}}$ Umuarama & 1 & 0,6 & - & - & 2 & 1,3 & 3 & 0,6 \\
\hline $13^{\mathrm{a}}$ Cianorte & - & - & - & - & - & - & - & - \\
\hline 14 Paranavaí & - & - & 4 & 2,5 & 3 & 1,9 & 7 & 1,4 \\
\hline $15^{\mathrm{a}}$ Maringá & 3 & 1,8 & 4 & 2,5 & - & - & 7 & 1,4 \\
\hline 16 Apucarana & 3 & 1,8 & 1 & 0,6 & 5 & 3,2 & 9 & 1,9 \\
\hline $17^{\mathrm{a}}$ Londrina & 4 & 2,4 & 6 & 3,7 & 5 & 3,2 & 15 & 3,1 \\
\hline 18ª Cornélio Procópio & 2 & 1,2 & - & - & - & - & 2 & 0,4 \\
\hline 19a Jacarezinho & - & - & 3 & 1,8 & 2 & 1,3 & 5 & 1,0 \\
\hline $20^{a}$ Toledo & 2 & 1,2 & - & - & 2 & 1,3 & 4 & 0,8 \\
\hline 21ª Telêmaco Borba & 5 & 3,0 & 3 & 1,8 & 1 & 0,6 & 9 & 1,9 \\
\hline $22^{a}$ Ivaiporã & - & - & - & - & - & - & - & - \\
\hline
\end{tabular}


Na Tabela 3, observa-se um decréscimo no quantitativo dos diagnósticos realizados entre 2015 e 2017. As doenças contempladas pelo Capítulo II - neoplasias (28,1\%) e, o Capítulo IV - Doenças Endócrinas, Nutricionais e Metabólicas (24,0\%) da CID 10, foram prevalentes, seguidas por doenças do Capítulo X Doenças do Aparelho Respiratório (10,7\%).

Tabela 3 - Crianças e adolescentes internados segundo os diagnósticos - Capítulos da CID 10, serviço clínico pediátrico de hospital público de ensino. Curitiba - PR, 2015-2017.

\begin{tabular}{|c|c|c|c|c|c|c|c|c|}
\hline \multirow{2}{*}{ CID-10 } & \multicolumn{2}{|c|}{2015} & \multicolumn{2}{|c|}{2016} & \multicolumn{2}{|c|}{2017} & \multicolumn{2}{|c|}{ Total } \\
\hline & n & $\%^{* *}$ & n & $\%^{* *}$ & n & $\%^{* \star}$ & n & $\%^{*}$ \\
\hline Capítulo II - Neoplasias (tumores) & 50 & 21,4 & 60 & 28,7 & 82 & 37,4 & 186 & 28.1 \\
\hline $\begin{array}{l}\text { C64.99 - Neoplasia maligna do rim, exceto } \\
\text { pelve renal }\end{array}$ & 6 & 12,0 & 4 & 6,6 & 4 & 4,9 & 14 & 7,5 \\
\hline C71.0 - Cérebro, exceto lobos e ventrículos & 1 & 2,0 & 3 & 32,0 & 6 & 7,3 & 10 & 5,4 \\
\hline $\begin{array}{l}\text { C76.4 - Neoplasia maligna de membro } \\
\text { superior }\end{array}$ & 4 & 8,0 & 6 & 10,0 & 12 & 14,6 & 22 & 11,8 \\
\hline C91.0 - Leucemia linfoblástica aguda & 19 & 38,0 & 22 & 36,6 & 23 & 28,0 & 64 & 34,4 \\
\hline Outros diagnósticos ${ }^{\star \star *}$ & 20 & 40,0 & 25 & 41,6 & 37 & 25,1 & 76 & 40,8 \\
\hline $\begin{array}{l}\text { Capitulo IV - Doenças endócrinas, nutricionais } \\
\text { e metabólicas }\end{array}$ & 71 & 30,5 & 46 & 22,0 & 42 & 19,2 & 159 & 24,0 \\
\hline $\begin{array}{l}\text { E10.1 - Diabetes mellitus insulinodependente; } \\
\text { com cetoacidose }\end{array}$ & 22 & 31,0 & 18 & 39,1 & 15 & 35,7 & 55 & 34,6 \\
\hline $\begin{array}{l}\text { E10.9 - Diabetes mellitus insulinodependente; } \\
\text { sem complicações }\end{array}$ & 14 & 19,7 & 7 & 15,2 & 2 & 4,7 & 23 & 14,4 \\
\hline $\begin{array}{l}\text { E84.0 - Fibrose cística com manifestações } \\
\text { pulmonares }\end{array}$ & 11 & 15,5 & 10 & 21,7 & 7 & 16,6 & 28 & 17,6 \\
\hline $\begin{array}{l}\text { E84.8 - Fibrose cística com outras } \\
\text { manifestações }\end{array}$ & 2 & 2,8 & 3 & 6,5 & 12 & 28,6 & 17 & 10,7 \\
\hline Outros diagnósticos ${ }^{\star \star *}$ & 22 & 31,0 & 8 & 17,4 & 6 & 14,3 & 36 & 22,6 \\
\hline Capítulo VI - Doenças do sistema nervoso & 20 & 8,6 & 19 & 9,1 & 26 & 11,9 & 65 & 9,8 \\
\hline G40.9 - Epilepsia, não especificada & 6 & 30,0 & 1 & 5,2 & 9 & 34,6 & 16 & 24,6 \\
\hline Outros diagnósticos ${ }^{* * *}$ & 14 & 70,0 & 18 & 94,7 & 17 & 65.4 & 49 & 75,4 \\
\hline Capítulo X - Doenças do aparelho respiratório & 31 & 13,3 & 25 & 11,9 & 15 & 6,8 & 71 & 10,7 \\
\hline $\begin{array}{l}\text { J15.9 - Pneumonia bacteriana não } \\
\text { especificada }\end{array}$ & 5 & 16,1 & 3 & 12,0 & 4 & 26,6 & 12 & 16,9 \\
\hline J96.0 - Insuficiência respiratória aguda & 13 & 41,9 & 15 & 60,0 & 7 & 46,6 & 35 & 49,3 \\
\hline Outros diagnósticos ${ }^{* \star *}$ & 13 & 41,9 & 7 & 28,0 & 4 & 26,6 & 24 & 33,8 \\
\hline $\begin{array}{l}\text { Capítulo XXI - Fatores que influenciam o } \\
\text { estado de saúde e o contato com os serviços } \\
\text { de saúde }\end{array}$ & 4 & 1,7 & 10 & 4,8 & 7 & 3,2 & 21 & 3.1 \\
\hline $\begin{array}{l}\text { Z03.9 - Observação por suspeita de doença } \\
\text { ou afecção não especificada }\end{array}$ & 4 & 10,0 & 9 & 90,0 & 5 & 71,4 & 18 & 85,7 \\
\hline Outros diagnósticos ${ }^{\star \star *}$ & - & - & 1 & 10,0 & 2 & 28,6 & 3 & 14,3 \\
\hline Outros capítulos ${ }^{* * * *}$ & 57 & 24,4 & 49 & 23.4 & 47 & 21.4 & 159 & 24,0 \\
\hline Total & 233 & 100 & 209 & 100 & 219 & 100 & 661 & 100 \\
\hline
\end{tabular}

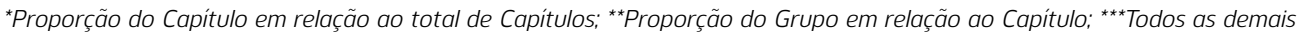
subcategorias relativas ao Capítulo, com ocorrência menor de 10 pessoas; ****Junção de 14 Capítulos. Fonte: Dados disponibilizados pelo Sistema de Informação Hospitalar. Elaborado pelos autores (2018). 
A Tabela 4 apresenta os resultados correspondentes ao tempo de internação em dias. Em 2015, as crianças e os adolescentes permaneceram internados de 0 a 43 dias; em 2016, houve uma redução para até 34 dias; voltando a elevar-se em 2017, para até 42 dias. Durante este período, a mediana do tempo de internação foi de 2 dias em 2015, de 3 dias em 2016, e de 4 dias em 2017, discreta variação.

Tabela 4 - Crianças e adolescentes internados segundo o tempo de internação (em dias), serviço clínico pediátrico de hospital público de ensino. Curitiba - PR, 2015-2017.

\begin{tabular}{lccc}
\hline Tempo de Internação (dias) & $\mathbf{2 0 1 5}$ & $\mathbf{2 0 1 6}$ & $\mathbf{2 0 1 7}$ \\
\hline Máximo & 43,00 & 34,00 & 42,00 \\
Mínimo & 0 & 0 & 0 \\
Média & 4,93 & 5,23 & 5,85 \\
Mediana & 2,00 & 3,00 & 4,00 \\
\hline
\end{tabular}

Fonte: Dados disponibilizados pelo Sistema de Informação Hospitalar. Elaborado pelos autores (2018).

Como mostra a Figura 1, segundo o mês das internações por ano, em 2015 houve prevalência das internações em agosto $(n=21)$ e setembro $(n=20)$. Já em 2016, janeiro e abril foram os meses prevalentes ( $n=18)$; e o mês de junho com menor quantitativo de internamentos. Em 2017, os meses de outubro e maio predominaram, destacando que setembro foi o mês que houve menos internações ( $n=5)$, diferença relevante quando comparado com 2015.

Figura 1 - Número de crianças e adolescentes internados segundo mês e ano de internação, serviço clínico pediátrico de hospital público de ensino. Curitiba - PR, 2015-2017.

$$
25
$$

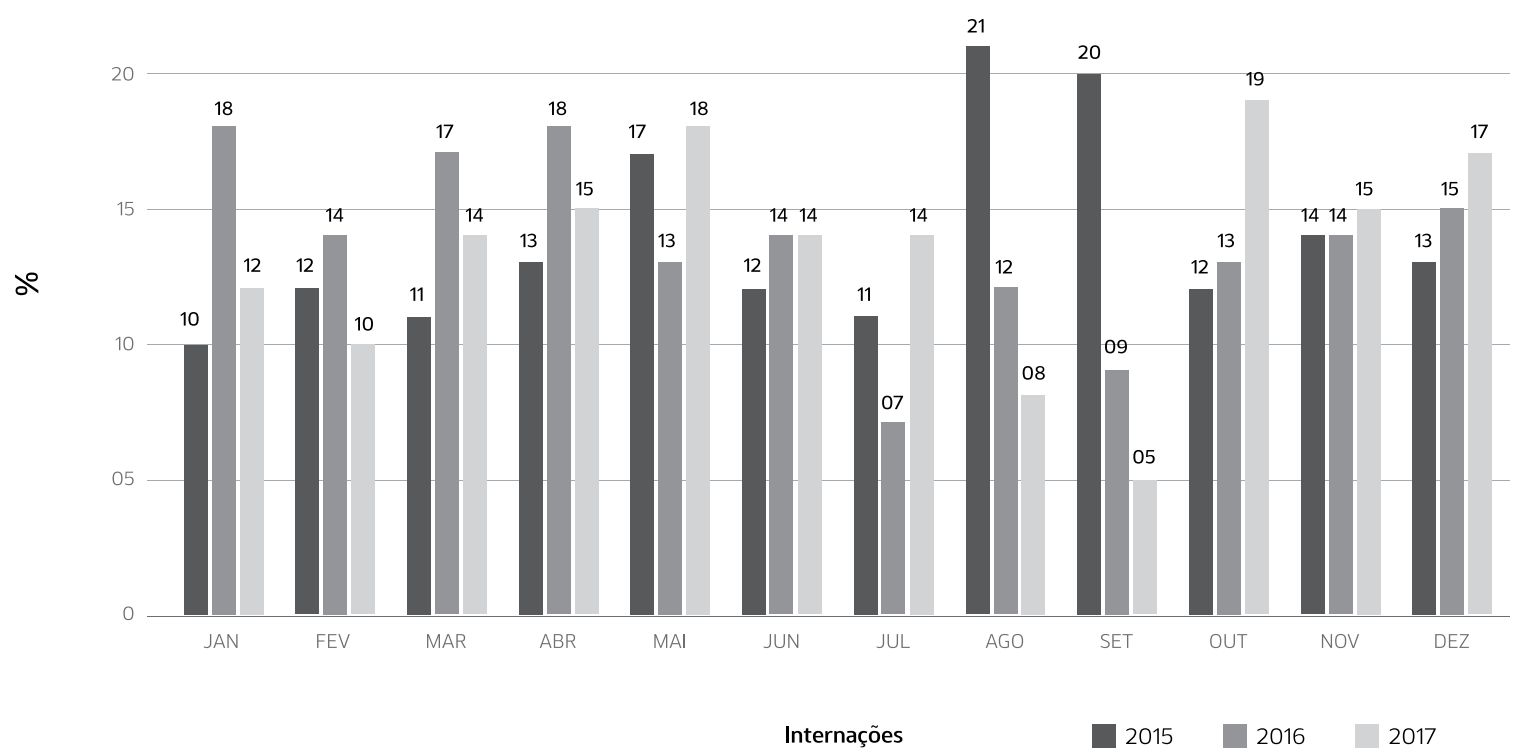

Fonte: Dados disponibilizados pelo Sistema de Informação Hospitalar. Elaborado pelos autores (2018). 
Destaca-se, entre os meses de fevereiro a abril, uma curva ascendente semelhante às internações de 2016 e 2017. O mesmo fenômeno aparece também em maio de 2015 e 2017. Pode-se apontar os meses de agosto e setembro como os mais discrepantes nesta análise, tendo em vista que em 2015 despontaram com os números mais elevados de internações, e em 2017 foram os mais baixos.

\section{DISCUSSÃO}

Convergente aos resultados aqui apresentados, o Instituto Brasileiro de Geografia e Estatística (IBGE), em 2010, registrou que a distribuição da população apresentou pequena predominância feminina (51,0\%) em nível nacional"1.

As crianças em idade pré-escolar (1 a 4 anos) e escolar ( 5 a 11 anos) foram as que mais necessitaram de internações, indicando que estas fases requerem mais cuidados intermediários de saúde, pois possuem características singulares de ordem fisiológica, comportamental e de exposição a riscos frente às ameaças do ambiente. Consequentemente, há reflexo na elevação da prevalência de infecções e outras doenças da infância como exemplo: diarreia, asma, cânceres e distúrbios endócrinos ${ }^{12}$.

$\mathrm{O}$ alto número de internações de pacientes procedentes de municípios que compõem a $2^{\mathrm{a}}$ Regional de Saúde é explicado por Curitiba ser o município-sede da mesma, e ser reconhecido como um polo médicoassistencial e educacional, no qual se localiza o hospital de ensino cenário do estudo. O Paraná possui Macro e Microrregiões de Saúde, cujos municípios participantes devem atender um número mínimo de procedimentos de média complexidade. Como primeiro nível de referência intermunicipal, Curitiba compõe exclusivamente uma Microrregião ${ }^{13}$, de maneira que o número de internações na Clínica Pediátrica pode expressar esta condição de média complexidade.

É interessante ressaltar que, embora haja na mesma estrutura do serviço pediátrico em estudo seis leitos destinados às crianças com problemas hematológicos e oncológicos, cerca de $28 \%$ das internações ocorridas por estes diagnósticos ocuparam os seis leitos que seriam para as demais especialidades clínicas. Trata-se de percentual significativo de crianças com Causa Básica da internação relacionada ao Capítulo II Neoplasias.

Segundo o Global Cancer Observatory - Cancer Today, da International Agency of Research on Cancer da WHO (World Health Organization), em 2018, a estimativa da taxa de incidência de câncer na faixa etária de 0 - 19 anos, por 100.000 habitantes da mesma faixa etária, variou nos continentes e países. Estas estimativas figuraram acima de 15,4 em alguns países, como exemplo, o Brasil, Canadá, Estados Unidos e parte da Europa - destacando-se a Bélgica, Canadá e Austrália, com taxas acima de 20,0/100.000. Já as menores de 6.4 estão registradas para alguns países da África, como Serra Leone, Guiné, Guiné Bissau, Libéria, Côte d'llvoire, Camarão, Angola e Namíbia, assim como em alguns da Ásia Central: Cazaquistão, Mongólia e Uzbequistão ${ }^{14}$. E as informações do Instituto Nacional do Câncer (INCA), de 2018, apontaram a incidência de 12.500 novos casos no Brasil, ou seja, incidentes de neoplasias infantis ${ }^{15}$.

O cenário com evidências incontestáveis da necessidade de (re) estruturação dos serviços de saúde pediátricos, com elevação dos leitos hospitalares exclusivos para diagnósticos e tratamento do câncer infantil, que possam contar com estrutura física e técnico-profissional de qualidade. Acredita-se que este provimento é necessário para favorecer o diagnóstico precoce, ou melhor dizendo, em tempo oportuno, o qual possibilite tratamentos menos agressivos, mais eficazes, ou seja, com maiores chances de cura ${ }^{16}$. Sob esta ótica, a Organização Mundial da Saúde, em 2018, anunciou uma iniciativa inovadora denominada Global Initiative for 
Childhood Cancer, cujo objetivo principal é elevar as taxas de sobrevivência até 2030 para 60\%. Isso significa a duplicação da taxa de cura de crianças em todo o mundo, com especial atenção às crianças de países de renda baixa e média, pois elas têm quatro vezes mais chance de morrerem¹.

Contemplaram a segunda e a terceira principais causas de internação, respectivamente, as doenças endócrinas, nutricionais e metabólicas, e as doenças do aparelho respiratório. Achados corroborados por um estudo realizado em 2015, em Fortaleza, a Diabetes Mellitus tipo 1 foi prevalente do Capítulo IV (16,8\%), seguida pelos problemas do aparelho respiratório (Capítulo X) com 12,6\% ${ }^{18}$.

Os Capítulos IV e X englobam doenças classificadas como Internações por Condições Sensíveis à Atenção Primária (ICSAPS), ou seja, sinalizam a fragilidade da assistência disponibilizada pela Atenção Primária à Saúde, sobretudo por haver protocolos de tratamento amplamente difundidos para tais. Este conceito implica também na consideração de que o atendimento hospitalar onera os gastos públicos e expõe a criança e adolescente aos riscos para maior morbidade ${ }^{18}$. Em 2012, um estudo no Maranhão apontou para a proporção das Internações por Condições Sensiveis à Atenção Primária, que foi 17\%19 inferior ao identificado pelo presente estudo (34,7\%).

Em relação ao tempo de internação identificado (de 0 a 43 dias, com mediana de 3 a 4 dias), os dados do estudo de Fortaleza são discordantes, pois as internações variaram de 1 a 70 dias com média de 10 dias $^{18}$. Os autores afirmam que o maior intervalo de dias de internação certamente relacionam-se com o atendimento de doentes crônicos ${ }^{18,20}$.

Quanto à sazonalidade das internações, houve maior proporção em cinco meses do ano, possivelmente relacionadas com diagnósticos de infecções respiratórias, devido a Curitiba e região apresentarem importante instabilidade climática, com início em março, que se estende até agosto. Já outubro sinaliza o início da primavera, manifestando-se os problemas respiratórios relacionados à alergia, devido à polinização das plantas, tempo seco e maior poluição ambiental ${ }^{21-22}$.

A alta médica foi o desfecho prevalente das internações estudadas. Mediante a melhora clínica a assistência hospitalar é interrompida, e a continuidade dos cuidados e uso de medicamentos específicos ocorre em âmbito hospitalar ${ }^{20}$.

Nesta perspectiva, destaca-se a relevância da atuação da equipe multiprofissional da área da saúde especialmente os enfermeiros, devido à atuação frente ao preparo educativo da criança e família para a alta hospitalar. Em estudo realizado na unidade pediátrica de um hospital-escola de São Paulo, em 2016, foi apresentada a importância da orientação feita pelos profissionais da saúde após a alta das crianças, a fim de estabelecer continuidade e qualidade dos cuidados de forma integral, transcendendo o contexto da doença, vivenciado no ambiente hospitalar. Este estudo ressalta, ainda, a responsabilidade do enfermeiro para esta atribuição, pois é o gestor do cuidado ${ }^{23}$.

Ademais, é evidenciada a importância da Sistematização da Assistência de Enfermagem em seu sentido mais amplo, que contempla ferramentas de organização estrutural, física e humana, bem como do Processo de Enfermagem, que propicia o cuidado individualizado e especializado ${ }^{24}$. Portanto, as características das internações de um serviço clínico pediátrico de um hospital de ensino, referência para o alto risco, além do potencial de subsidiar tomadas de decisão pelos gestores da unidade, pode apontar a necessidade de estratégias de previsão para recursos materiais e humanos; para o gerenciamento dos leitos especializados, sobretudo os oncológicos e do cuidado à saúde das crianças, adolescentes e acompanhantes. Além de replicável, o estudo favorece a comparabilidade com outros cenários nacionais e internacionais, contribuindo para o incremento de estudos nesta perspectiva. 
E ainda, este estudo possibilita apontar para a necessidade de qualificação da Atenção Primária em Saúde, nos municípios da $2^{\text {a }}$ Regional de Saúde, e poderá contribuir com a análise da Linha de Cuidado Infantil, no Paraná. Todos os aspectos contributivos têm a visão da garantia do acesso ao serviço hospitalar público e, da assistência segura, humanizada e de qualidade às crianças, aos adolescentes e seus familiares³.

\section{CONCLUSÃO}

A caracterização das internações permite a formação de subsídios a serem utilizados pela gestão na tomada de decisão. Com isso, favorece o planejamento da assistência à saúde pediátrica, contribuindo para a sua realização com maior qualidade. E também propõe novas pesquisas voltadas para a qualidade de vida destas crianças e seu desenvolvimento.

\section{AGRADECIMENTOS}

Agradecemos ao Complexo Hospital de Clínicas pela receptividade à proposta de estudo; ao Programa de Iniciação Científica - PIBIC, da Universidade Federal do Paraná; e ao CNPq - Conselho Nacional de Desenvolvimento Científico e Tecnológico, pela Bolsa recebida ao longo de um ano no PIBIC/UFPR.

\section{REFERÊNCIAS}

1. Brasil. Portaria $n^{\circ}$ 1.130, de 5 de agosto de 2015. Dispõe sobre a Política Nacional de Atenção Integral à Saúde da Criança no âmbito do Sistema Único de Saúde. [Cited 2020 Mar 22]. Disponível em:https://bit.ly/33KduXi.

2. Brasil. Lei n 8.069, de 13 de julho de 1990. Dispõe sobre o Estatuto da Criança e do Adolescente e dá outras providências. Presidência da República. Casa Civil. Subchefia para Assuntos Jurídicos. Brasília, DF. 1990.

3. Pedraza EF, Araujo EMN. Internações das crianças brasileiras menores de cinco anos: revisão sistemática da literatura. Epidemiol Serv Saúde. [Internet] 2017:26(1):169-82. [Cited em 2020 Mar 22]. DOI: 10.5123/s1679-49742017000100018.

4. Cruz DALM, Pimenta CAM. Prática baseada em evidências, aplicada ao raciocínio diagnóstico.Rev Latino-am Enfermagem. [Internet]. 2005;13(3):415-22. [Cited em 2020Mar22]. DOI: 10.1590/S0104-11692005000300017.

5. Granzotto JA, Mota DM, Vecchi AA, Santos EO, Gonçalves ER, Silva JBY et al. Características sócio demográficas maternas e perfil das crianças internadas em um hospital do sul do Brasil. Rev Enferm UFSM. [Internet] 2014:4(1):97-104. [Cited em 2020 Mar 22]. DOl: $10.5902 / 217976928466$.

6. Aragão J. Introdução aos estudos quantitativos utilizados em pesquisas científicas. Revista Práxis. 2011:3(6)11. [Cited em 2020 Mar 22] DOI: 10.25119/praxis-3-6-566.

7. Malta M, Cardoso LO, Bastos, FI, Magnanini MMF, Silva CMFP. Iniciativa STROBE: subsídios para a comunicação de estudos observacionais. Rev Saúde Pública 2010:44(3):559-65. [Cited em 2020 Mar 22]. DOI: 10.1590/S0034-89102010000300021.

8. CHC-UFPR-EBSERH. Complexo Hospital de Clínicas. Universidade Federal do Paraná. Empresa Brasileira de Serviços Hospitalares. [Homepage on the internet] Página oficial. 2018. [Cited em 2020 Mar 22]. Disponivel em: https://bit.ly/2U8iGB4.

9. Organização Mundial de Saúde. CID-10; tradução Centro Colaborador da OMS para a Classificação de Doenças em Português. 9. ed. São Paulo: Editora da Universidade de São Paulo. 2003.

10. Brasil. Resolução $n^{\circ}$ 466, de 12 de dezembro de 2012. [Internet]. Institui Resolução que trata de pesquisas e testes com seres humanos. [Cited em 2020 Mar 22]. Disponivel em: https://bit.ly/2y0eASU. 
11. Brasil. Instituto Brasileiro de Geografia e Estatística. Distribuição da População por sexo segundo Unidades da Federação. 2010.

12. Perlroth NR, Branco CWC. Current knowledge of environmental exposure in children during the sensitive developmental periods. J Pediatr. [Internet] 2017;93:17-7. [Cited em 2020 Mar 22].Doi: 10.1016/j.jped.2016.07.002.

13. Secretaria de Saúde do Estado Paraná. Plano diretor de regionalização: hierarquização e regionalização da assistência à saúde, no estado do Paraná. [Citedem 2020 Mar 22]. Disponível em: https://bit.ly/3dn8Tys.

14. WHO. World Health Organization. International Agency of Research on Cancer. Global CancerObservatory - Cancer Today. [Internet] 2019. [Cited em 2020 Mar 22]. Disponivel em:https://bit.ly/3dkeRjN

15. INCA. Instituto Nacional de Câncer (Brasil). Câncer Infantil. [Internet]. 2017. [Cited em 2020 Mar 22]. Disponível em: https://bit.ly/3bhsCxF.

16. Delfino CTA, Ferreira WFS, Oliveira EC. Dutra DA. Câncer infantil: Atribuições da enfermagem em cuidado paliativo. Rev Saude Desenvol. [Internet]. 2018;12(10). [Cited em 2020 Mar 22]. Disponivel em: https://bit.ly/2wjRaYj.

17. WHO. World Health Organization. Global Initiative for Childhood Cancer. [Internet]. 2019. [Cited em 2020Mar 22]. Disponivel em: https:// bit.ly/2wwPcDV.

18. Parente JSM, Silva FRA. Perfil clínico epidemiológico dos pacientes internados na clínica pediátrica de um hospital universitário. Rev Med UFC. 2017:57(1):10-4. [Cited em 2020 Mar 22]. DOI: 10.20513/2447-6595.2017v57n1p10-14.

19. Lima RCDSM, Gama MEA, Lima RDSM. Condições sensiveis à atenção primária em hospital de referência pediátrica no Maranhão. Rev Pesq Saúde. [Internet] 2017:18(2):97-101. [Cited em 2020 Mar 22]. Disponível em: https://bit.ly/2Urr4um

20. Ramos SA. A influência do planeamento da Alta Hospitalar no número de dias de internamento do doente. (Dissertação). Universidade Lusófona de Humanidades e Tecnologias. Escola de Ciências e Tecnologias da Saúde. Lisboa. 2015.

21. Silveira RB, Rocha GS, Alves MPA. Contabilização das ondas de frio em Curitiba, Paraná. Eixo: Climatologia em diferentes níveis escalares: mudanças e variabilidades. Instituto de Geociências da Unicamp. Campinas - SP. 2017. [Cited em 2020 Mar 22]. DOI: 10.20396/sbgfa. v1i2017.2434.

22. Steven GG. Una pequeña dosis de contaminación del aire. Una introducción a los efectos de la contaminación del aire em la salud. In: . Toxicología de la contaminación del aire. Chapter. 2016

23. Vieira MM. O cuidado à criança após a alta hospitalar. Rev Bras Saude Func. [Internet] 2016:1(3):12. [Cited em 2020 Mar 22]. Disponível em: https://bit.ly/33Atsml.

24. Soares MI, Resck ZMR, Terra FS, Camelo SHH. Systematization of nursing care: challenges and features to nurses in the care management. Escola Anna Nery RevEnferm. 2015;19(1). [Cited em 2020 Mar 22]. DOI: 10.5935/1414-8145.20150007. 